\title{
Lipid profile with paternal history of coronary heart disease before age 40
}

Tzvy Bistritzer, Lorena Rosenzweig, Joseph Barr, Sonica Mayer, Eliezer Lahat, Hedy Faibel, Zwi Schlesinger, Mordechai Aladjem

\begin{abstract}
Serum lipids were measured in children and their parents from 40 families in which the father had a myocardial infarction or coronary heart disease (CHD) before age 40 years. The relationship between physical activity and serum lipid concentrations in the children was also evaluated.
\end{abstract}

Twenty six men had one or more abnormal lipid value (in $\mathrm{mmol} / \mathrm{l}$ ): total venous cholesterol (TVC) $>6 \cdot 24$, triglycerides $>2 \cdot 55$, low density lipoprotein cholesterol (LDL-C) $>4.42$, or high density lipoprotein cholesterol (HDL-C) $<0.91$. There were 15 spouses with significant hyperlipidaemia (values above). In the 107 children examined, TVC mean (SD) was 4.68 (1.17), triglycerides $1.4(0.8)$, LDL-C $3.0 \quad(1 \cdot 0)$, and HDL-C $1.18 \quad(0 \cdot 28)$. Altogether $42 \%$ of the children had significant hyperlipidaemia. No significant correlation was found between the degree of physical activity of the children and their LDL-C and TVC concentrations. However, a significant positive correlation was found between the degree of physical activity and HDL-C and a significant negative one with triglyceride concentrations.

It is concluded that screening the progeny of young CHD patients is highly productive in identifying young people at excessive risk for future CHD. The data also suggest that promoting high degrees of activity among these children may have a positive influence on risk factors for adult onset CHD.

(Arch Dis Child 1995; 73: 62-65)

Assaf Harofeh Medical the Sackler Faculty of Medicine, Tel Aviv University, Israel, Department of

Paediatrics

T Bistritzer

L Rosenzweig

J Barr

E Lahat

M Aladjem

Department of

Biochemical Pathology

$S$ Mayer

Department of

Cardiology

H Faibel

Z Schlesinger

Correspondence to: Dr T Bistritzer, Department of Paediatrics 'B', Assaf Harofeh Medical Centre Zerifin 70300, Israel.

Accepted 26 February 1995 Health, USA, recommended a blood cholesterol test for children whose parents, grandparents, or first degree relatives had hypercholesterolaemia or premature coronary heart disease (CHD) $(<50$ years of age in men, $<60$ years of age in women). ${ }^{5}$ Many surveys examining the lipoproteins in the progeny of young men with $\mathrm{CHD}$ have followed this recommendation. ${ }^{126-8}$

A relationship between physical activity and increased concentrations of high density lipoprotein cholesterol (HDL-C) in both young ${ }^{9}$ and middle aged men has been demonstrated. ${ }^{10}$ However, similar studies performed in young children show conflicting data. ${ }^{11} 12$ The present investigation was undertaken to study the lipoprotein fractions in children whose fathers had myocardial infarction or CHD before the age of 40 years. The lipoprotein fractions were analysed in the parents as well. The relationship between physical activity and serum lipid concentrations in these children was also evaluated.

\section{Subjects and methods}

Children from 40 families in which the father had a myocardial infarction or CHD before the age of 40 years and were treated in our hospital, were invited to attend the paediatric clinic for a physical examination and serum lipid determination. We included in the study only adults with CHD and angiography proved coronary artery stenosis of more than $70 \%$ of one or more major branches. The children were examined by the same physician throughout the study. None of the children had any symptoms and signs of secondary hypercholesterolaemia due to hypothyroidism, nephrotic syndrome, liver disease, or diabetes mellitus. None were receiving any continuous medical treatment. Body weight, height, and blood pressure were recorded. Body mass index, weight/height ${ }^{2}$, was used as an obesity index. Satisfactory dietary intake information was difficult to obtain; however, $80 \%$ of the families did not adhere to a low saturated fat-low cholesterol diet. After a 12 hour fast, total venous cholesterol (TVC), triglycerides, and HDL-C were determined in both children and parents. LDL-C was calculated using the standard formula: LDL$\mathrm{C}=$ TVC minus HDL-C minus triglycerides $/ 5$. The blood tests were performed using an IL Monarch analyser. Lipid values were reproducible within $5 \%$ of true value and the same values were reproducible within $4 \%$ of a standard sample. The time interval between the coronary event and the evaluation of the lipid profile was one to two years. We obtained parental histories of additional cardiovascular risk factors such as diabetes, hypertension, and smoking. 
Table 1 Lipoprotein concentrations (mmoll) in 34 men with early CHD [number in parenthesis shows number of men studied]

\begin{tabular}{llll}
\hline & Acceptable & Borderline & High \\
\hline Total cholesterol & $<5 \cdot 20[7]$ & $5 \cdot 20-6 \cdot 24[10]$ & $>6 \cdot 24[17]$ \\
Triglycerides & $<1.95[8]$ & $1 \cdot 95-2 \cdot 55[10]$ & $>2 \cdot 55[16]$ \\
LDL-C & $<3.38[8]$ & $3 \cdot 38-4 \cdot 42[10]$ & $>4 \cdot 42[16]$ \\
HDL-C & $>1.17[9]$ & $0.91-1 \cdot 17[14]$ & $<0.91[11]$ \\
\hline
\end{tabular}

Table 2 Lipoprotein concentrations ( $\mathrm{mmol} / \mathrm{l}$ ) in 40 spouses of 34 men with early CHD [number in parenthesis shows number of spouses studied]

\begin{tabular}{llll}
\hline & Acceptable & Borderline & High \\
\hline Total cholesterol & $<5 \cdot 20[19]$ & $5 \cdot 20-6 \cdot 24[13]$ & $>6.24[8]$ \\
Triglycerides & $<1.95[29]$ & $1.95-2 \cdot 55[3]$ & $>2.55[8]$ \\
LDL-C & $<3.38[17]$ & $3 \cdot 38-4 \cdot 42[17]$ & $>4.42[6]$ \\
HDL-C & $>1.17[27]$ & $0.91-1 \cdot 17[11]$ & $<0.91[2]$ \\
\hline
\end{tabular}

Children were classified into four groups according to their physical activity including gymnastics, swimming, soccer, basketball, and swimming. In group 1 there was no physical activity (16 children); group $2,<3.5$ hours/ week of physical activity ( 42 children); group $3,3 \cdot 5-7$ hours/week of physical activity ( 31 children); and group $4,>7$ hours/week physical activity (18 children).

The study was approved by the research and human ethics committees of our hospital.

\section{STATISTICAL METHODS}

Results are presented as the mean (SD) in $\mathrm{mmol} / \mathrm{l}$. The influence of physical activity on lipid concentrations was assessed by $\chi^{2}$ and Wilcoxon rank tests; $p$ values $<0.05$ were considered significant. The relationship between TVC and LDL-C concentrations was evaluated by linear regression analysis.

\section{Results}

Three fathers from 40 families had died from myocardial infarction and three fathers refused to participate. The age of the 34 fathers tested for hyperlipidaemia was $38(2 \cdot 1)$ (range 31-41) years. Twenty nine patients had a mycocardial infarction: 10 of the anterior wall, 16 of the inferior wall, one of the lateral wall, and two of the posterior wall; five patients suffered from severe CHD. The lipid profile of the 34 men is summarised in table 1. Only eight patients had normal lipid concentrations. Twenty six men $(77 \%)$ had one or more abnormal value of the following (in $\mathrm{mmol} / \mathrm{l}$ )

Table 3 Lipoprotein concentrations (mmoll) in 107 children whose fathers had early $C H D$ and lipoprotein concentrations measured $(n=34)$ [number in parenthesis signifies the number of children studied]

\begin{tabular}{|c|c|c|c|c|}
\hline & Acceptable & Borderline & $\begin{array}{l}\text { High or low } \\
\text { for } H D L-C\end{array}$ & Very high \\
\hline $\begin{array}{l}\text { Total cholesterol } \\
\text { Mean (SD) } \\
\text { Triglycerides } \\
\text { Mean (SD) } \\
\text { LDL-C } \\
\text { Mean (SD) } \\
\text { HDL-C } \\
\text { Mean (SD) }\end{array}$ & $\begin{aligned}<4.42[46] \\
3.80(0.49) \\
<1.50[70] \\
1.00(0.24) \\
<2.60[37] \\
2.10(0.38) \\
>1.17[49] \\
1.46(0.18)\end{aligned}$ & $\begin{array}{l}4 \cdot 42-5 \cdot 17[35] \\
4 \cdot 76(0 \cdot 23) \\
1 \cdot 50-1 \cdot 92[22] \\
1 \cdot 68(0 \cdot 13) \\
2 \cdot 60-3 \cdot 35[43] \\
3 \cdot 00(0 \cdot 21) \\
0 \cdot 91-1 \cdot 17[40] \\
1 \cdot 00(0 \cdot 08)\end{array}$ & $\begin{array}{l}5 \cdot 20-6 \cdot 24[18] \\
5 \cdot 67(0 \cdot 36) \\
1.95-2 \cdot 55[11] \\
2 \cdot 16(0 \cdot 11) \\
3 \cdot 38-4 \cdot 16[12] \\
3 \cdot 60(0 \cdot 16) \\
<0.91[18] \\
0.80(0.05)\end{array}$ & $\begin{array}{r}>6.24[8] \\
7.56(0 \cdot 80) \\
>2.55[4] \\
4.50(1 \cdot 70) \\
>4 \cdot 16[15] \\
4.80(0 \cdot 80)\end{array}$ \\
\hline
\end{tabular}

TVC $>6 \cdot 24$, triglycerides $>2 \cdot 55$, LDL-C $>4.42$, or HDL-C $<0.91$. TVC concentration in the whole group was $6.50(1.50)$, triglycerides $2.80(1 \cdot 35)$, LDL-C $4.62(1 \cdot 40)$, and HDL-C $1 \cdot 10(0 \cdot 28)$. There was no significant difference between the lipid profile in the men with anterior compared with inferior wall myocardial infarction. Examining coronary risk factors in these 34 men, 27 of them (79\%) were heavy smokers (more than 20 cigarettes/day for more than three years preceding the coronary event), 22 had a positive coronary family history, seven had hypertension, and six were obese (body mass index $>30 \mathrm{~kg} / \mathrm{m}^{2}$ ). There was no correlation between the body mass index values on one hand and the TVC, triglycerides, $\mathrm{LDL}-\mathrm{C}$, and $\mathrm{HDL}-\mathrm{C}$ values on the other.

TVC concentrations in the 40 spouses were (in $\mathrm{mmol} / \mathrm{l}$ ) $5.46(1.35)$, triglycerides 1.65 $(0 \cdot 78)$, LDL-C $3 \cdot 60(1 \cdot 20)$, and HDL-C 1.30 $(0 \cdot 30)$. The lipid profile of the 40 spouses is summarised in table 2 . There were 15 spouses $(38 \%)$ with significant hyperlipidaemia: TVC $>6 \cdot 24$, triglycerides $>2 \cdot 55$, LDL-C $>4 \cdot 42$, or HDL-C $<0.91$ or in any combination. No spouse had CHD. Six of the mothers were obese (body mass index $>30 \mathrm{~kg} / \mathrm{m}^{2}$ ). There was no correlation between the body mass index values on one hand and the TVC, triglycerides, LDL-C, and HDL-C values on the other.

In the 34 families where fathers were tested for hyperlipidaemia, we identified 107 children aged $12(4 \cdot 6)$ (range 2-20) years. There were 59 boys and 48 girls. TVC concentration was (in $\mathrm{mmol} / \mathrm{l}$ ) $4.68(1 \cdot 17)$, triglycerides 1.40 $(0 \cdot 80)$, LDL-C $3.00(1 \cdot 00)$, and HDL-C 1.18 $(0 \cdot 28)$. The lipid profile of the children is summarised in table 3 . Altogether $42 \%$ of the children had significant hyperlipidaemia (above the 95th centile): TVC $>5 \cdot 20$, LDL-C $>3.38$, triglycerides $>1.95$ or HDL-C $<0.91$ or in any combination. In 26 families, at least one child in every family was found to have a lipoprotein abnormality. In eight families, both parents had lipoprotein abnormalities, in 18 families only the father, and in three families only the mother was found to have hyperlipidaemia. Only one child was obese (body mass index $>27 \mathrm{~kg} / \mathrm{m}^{2}$ ). A positive correlation was found between TVC and LDL-C levels $(r=0.8, \mathrm{p}<0.001)$. No correlation was found between the age and sex of the children and lipid concentrations.

No significant correlation was found between the degree of physical activity of the children and their LDL-C and TVC levels (figure). However, a significant positive correlation was found between the degree of physical activity and HDL-C $(p<0.05)$ and a significant negative one with triglyceride concentrations $(p<0.05)$ (figure). There was a strong association between the HDL-C in children whose physical activity corresponded to groups 2-4 and their fathers. However, no such association was found in the children with no physical activity (group 1). In those children, the lack of physical activity was the main reason for their low HDL-C concentration. 


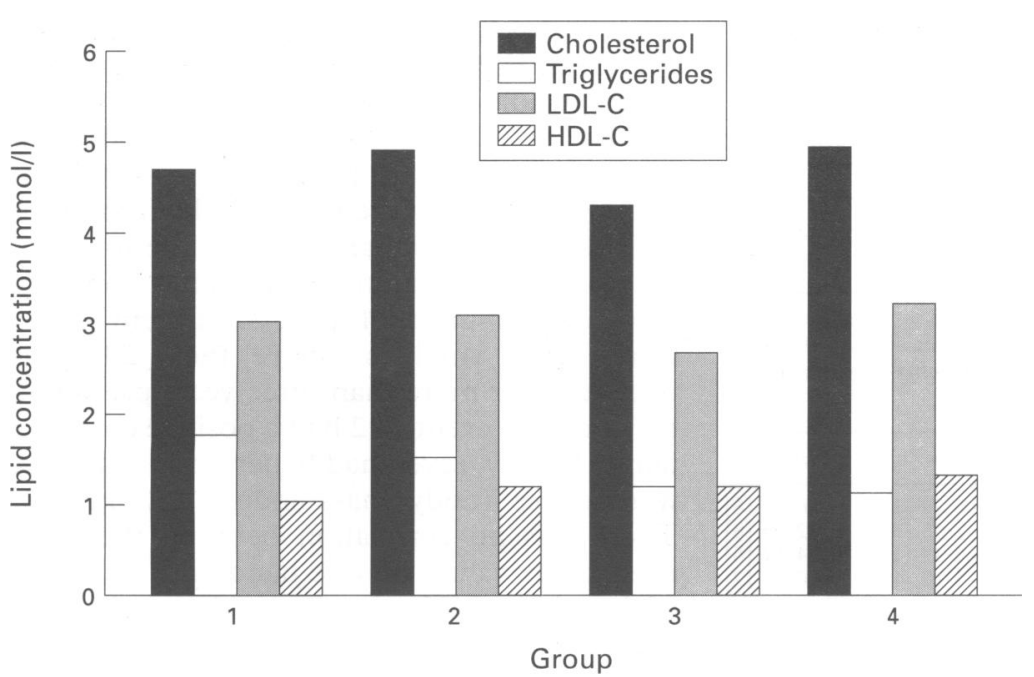

Distribution of the lipid concentrations in the four groups of children according to their degree of physical activity (group 1, no physical activity; group 4, the highest level of activity, see methods section).

\section{Discussion}

There are only a few studies ${ }^{13} 14$ which examined the lipid profile in children from kindreds with premature parental myocardial infarction. In our study, we confirmed the findings of a high incidence of raised total cholesterol and triglyceride in the progeny of young men (under 40 years) with CHD.

We performed a cholesterol routine surveillance study of 809 children 6 to 14 years of age chosen at random. The mean (SD) cholesterol concentration was $3 \cdot 74(0 \cdot 6) \mathrm{mmol} / \mathrm{l}$ (unpublished data). The mean cholesterol concentrations in the present study, which represents a high risk group of children for the development of CHD, was significantly higher, 4.68 $(1.25) \mathrm{mmol} / \mathrm{l}(\mathrm{p}<0.001)$. In addition, we have also shown that abnormalities of the lipoprotein fractions with raised LDL-C and diminished HDL-C concentrations are frequently present in these children. Forty two per cent of the offspring had an abnormality of total lipids or lipoproteins. Prospective studies have clearly shown that raised plasma cholesterol is associated with an increased risk for the development of myocardial infarction or coronary artery disease. ${ }^{1516}$ Because of the significant correlation of total plasma cholesterol and LDL-C found in our study $(r=0 \cdot 8)$, LDL-C may serve as a strong indicator of $\mathrm{CHD}$.

We noted a higher incidence of hyperlipoproteinaemia $(77 \%)$ in young male survivors of coronary disease than in other studies. ${ }^{2} 1314$ Different arbitrary limits of normal lipid values as well as differing laboratory methodology, study design, and environmental differences between populations may influence the prevalence of hyperlipoproteinaemia among the various studies. Interestingly, no negative correlation was found in our study between body mass index and HDL-C values in both parents contrary to other studies. ${ }^{17} 18 \mathrm{~A}$ very high incidence of heavy smokers $(79 \%)$ was also found in the men with premature CHD. In the study of Nora et al the highest risk ratio for early onset ischaemic heart disease was associated with a positive family history for ischaemic heart disease. ${ }^{19}$
As with previous studies 112021 no correlation was found between the degree of physical activity and cholesterol or LDL-C concentrations. However, the degree of physical activity correlated inversely with HDL-C concentrations. Moreover, lack of physical activity was the main contributor to the low HDL-C, being more significant than genetic influence. Klesges et al found no significant relationships between physical activity and CHD risk variables in their group of preschool children, and suggested that the relationship between physical activity and health outcome variables may be weaker among children than adults. ${ }^{22}$ Our finding of $17 \%$ of children with low HDL-C concentrations in families with early onset CHD, emphasises the importance of physical activity and fitness among these high risk children in preventing adult onset $\mathrm{CHD}$. Confirming other studies, ${ }^{2324}$ we found that children with lower physical activity had higher triglyceride concentrations.

We conclude that screening the progeny of young coronary artery disease patients is highly productive in identifying young people at excessive risk for future coronary artery disease. Early identification of this young high risk population offers an opportunity for early initiation of preventive measures. We also found that cardiovascular fitness appears to have a positive influence on HDL-C and higher physical activity was associated with lower triglyceride concentrations. These data suggest that promoting high degrees of activity among children from a high risk group for CHD may have a positive influence on risk factors of adult onset CHD.

We are indebted to Mrs Anji Agajany and Mrs Jane Geva for their assistance in the preparation of this manuscript.

1 Lee J, Lauer RM, Clarke WR. Lipoproteins in the progeny of young men with coronary artery disease: children with increased risk. Pediatrics 1986; 78: 330-7.

2 Chase HP, O'Ouin RI, O'Brien D. Screening for hyperlipidemia in childhood. $\mathscr{f} A M A$ 1974; 230 : 1535-7.

3 Morrison JA, Khoury P, Laskarzewski PM, Mellies MJ Morrison JA, Khoury P, Laskarzewski PM, Mellies MJ,
Heinemeger R, Glueck CJ. Familial associations of lipids and lipoproteins in families of hypercholesterolemic probands. Atherosclerosis 1982; 2: 151-9.

4 Lauer RM, Lee J, Clarke WR. Factors affecting the relationship between childhood and adult cholesterol levels. The Muscatine study. Pediatrics 1988; 82: 309-18

5 Consensus Conference. Lowering blood cholesterol to prevent heart disease. $\mathcal{F} A M A$ 1985; 253: $2080-6$.

6 Larsson B, Vaara J. Cholesterol screening of seven-year-old children. How to identify children at risk. Acta Paediatr 1992; 81: 315-8.

7 Glueck CJ, Fallat RW, Tsang R, Buncher CR. Hyperlipidemia in progeny of parents with myocardial infarction before age 50. Am F Dis Child 1974; 127: 70-5

8 Sveger T, Fex G, Borfors N. Hyperlipidaemia in schoo children with family histories of premature coronary heart disease. Acta Paediatr Scand 1987; 76: 311-5.

9 Linder CW, DuRant RH. Exercise, serum lipids and cardiovascular disease risk factors in children. Pediatr Clin North Am 1982; 29: 1341-54.

10 DuRant RH, Linder CW, Mahoney OM. The relationship between habitual physical activity and high-density lipoprotein in white adolescents. $\mathcal{F}$ Adolesc Health Care 1983; 4: 235-40.

11 Hofman A, Walter HJ. The association between physical fitness and cardiovascular disease risk factors in children in a five-year follow-up study. Fournal of Epidemiology 1989; 18: 830-5.

12 Epstein LH, Kuller LH, Wing RR, Valoski A, McCarley J. The effect of weight control on lipid changes in obese children. Am f Dis Child 1989; 143: 545-7.

13 Tamir I, Bojanower Y, Levtow O, Heldenberg D, Dickerman Z, Werbin B. Serum lipids and lipoproteins in children from families with early coronary heart disease. Arch Dis Child 1972; 47: 808-10.

14 Heinle RA, Levy RI, Fredrickson DS, Gorlin R. Lipid and carbohydrate abnormalities in patients with angiographically documented coronary artery disease. $A m \mathcal{F}$ Cardiol 1969; 24: 178-86. 
15 Wissler RW. The emerging cellular pathobiology of atherosclerosis. Artery 1977; 5: 409-23.

16 Motulsky AG. The genetic hyperlipidemias. $N$ Engl $f$ Med 1976; 94: 823-7.

17 Goldbourt U, Yaari A, Cohen-Mandelzweig L, et al. Highdensity lipoprotein cholesterol correlation with biochemical, arthropometric, behavioural and clinical parametry in

18 Glueck CF, Taylor HL, Jacobs D, et al. Plasma high-density lipoprotein cholesterol: association with measurements of body mass. The lipid research clinics program study. Circulation 1982; 62: IV 62-9.

19 Nora J, Lortscher RH, Sprangler RD, Nora $A H$, Kimberling WJ. Genetic-epidemiologic study of earlyonset ischemic heart disease. Circulation 1980; 61: 503-8. 20 DuRant RH, Linder CW, Harkass JW, Gray RG. The rela- tionship between physical activity and serum lipids and lipoproteins in black children and adolescents. $\mathcal{F}$ Adolesc Health Care 1983; 4: 55-60. 21 Tholand WG

borland WG, Gilliam TB. Comparison of serum lipids between habitually high and low active preadolescent males. Med Sci Sports Exerc 1981; 13: 316-21.

22 Klesges RC, Haddock CK, Eck LH. A multimethod approach to the measurement of childhood physical activity and its relationship to blood pressure and body weight. f Pediatr 1990; 116: 883-93.

23 Epstein LH, Kuller LH, Wing RR, Valeski A, McCarley J. The effect of weight control on lipid changes in obese children. Am $\mathcal{F}$ Dis Child 1989; 143: 454-7.

24 Stewart KJ, Goldberg AP. Exercise, lipids and obesity in adolescents with parental history of coronary disease. American fournal of Health and Prevention 1992; 6: 430-6. 